\title{
Genome-Wide DNA Methylation Profiles in Renal Tumors of Various Histological Subtypes and Non-Tumorous Renal Tissues
}

\author{
Eri Arai $^{\mathrm{a}}$ Saori Wakai-Ushijima ${ }^{\mathrm{a}}$ Hiroyuki Fujimoto $^{\mathrm{d}}$ Fumie Hosoda $^{\mathrm{b}}$ \\ Tatsuhiro Shibata $^{b}$ Tadashi Kondo $^{c}$ Sana Yokoi $^{\mathrm{e}}$ Issei Imoto $^{\mathrm{e}}$ Johji Inazawa $^{\mathrm{e}}$ \\ Setsuo Hirohashi ${ }^{a}$ Yae Kanai ${ }^{a}$ \\ a Pathology Division, ${ }^{b}$ Cancer Genomics Project, and ${ }^{c}$ Proteome Bioinformatics Project, National Cancer Center \\ Research Institute, ${ }^{d}$ Urology Division, National Cancer Center Hospital, and ${ }^{\mathrm{e}}$ Department of Molecular \\ Cytogenetics, Medical Research Institute and School of Biomedical Science, Tokyo Medical and Dental University, \\ Tokyo, Japan
}

\section{Key Words}

Chromophobe renal cell carcinoma $\cdot$ Clear cell renal

cell carcinoma - DNA methylation - Oncocytoma •

Papillary renal cell carcinoma $\cdot$ Precancerous condition

\begin{abstract}
Objective: The aim of this study is to clarify genome-wide DNA methylation profiles in renal tumors of various histological subtypes. Methods: Bacterial artificial chromosome (BAC) array-based methylated CpG island amplification was performed using tissue samples of 17 patients with papillary renal cell carcinomas (RCCs), chromophobe RCCs and oncocytomas, and the results were compared with those from 51 patients with clear cell RCCs. Results: Unsupervised hierarchical clustering analysis based on DNA methylation status clustered type 1 and type 2 papillary RCCs into different subclasses. Although chromophobe RCCs and oncocytomas were clustered into the same subclass, the DNA methylation status of 21 BAC clones was able to discriminate chromophobe RCCs from oncocytomas. The number of BAC clones showing DNA methylation alteration in non-tumorous renal tissue from patients with chromophobe RCCs and oncocytomas was smaller than that from patients with clear cell RCCs.
\end{abstract}

Biphasic accumulation of DNA methylation alterations was observed in non-tumorous renal tissue from all 68 patients, and patients showing such alterations on more BAC clones had a poorer outcome than patients showing them on fewer BAC clones. Conclusions: DNA methylation profiles determining the histological subtypes of renal tumors developing in individual patients and/or patient outcome may be already established in non-tumorous renal tissue at the precancerous stage.

Copyright $\odot 2011$ S. Karger AG, Basel

\section{Introduction}

Accumulating evidence suggests that not only genetic but also epigenetic alterations play a significant role in human carcinogenesis. DNA methylation alterations are one of the most consistent epigenetic changes occurring during carcinogenesis in various organs: it is known that DNA hypomethylation results in chromosomal instability as a result of changes in chromatin structure, and that DNA hypermethylation of CpG islands silences tumorrelated genes in cooperation with histone modification [1-5].

\section{KARGER}

Fax +41613061234

E-Mail karger@karger.ch

www.karger.com
(C) 2011 S. Karger AG, Basel

$1015-2008 / 11 / 0781-0001 \$ 38.00 / 0$

Accessible online at:

www.karger.com/pat
Yae Kanai

Pathology Division, National Cancer Center Research Institute

5-1-1 Tsukiji, Chuo-ku

Tokyo 104-0045 (Japan)

Tel. +81 33542 2511, Fax +81 33248 2463, E-Mail ykanai@ ncc.go.jp 
Although the classification of renal tumors is based largely on histology, the World Health Organization classification has introduced genetic alterations as a hallmark corresponding to the histological subtypes of renal tumors, e.g. clear cell renal cell carcinomas (RCCs), the most common histological subtype, are characterized by loss of chromosome $3 p$ and inactivation of the VHL gene at 3p25.3 [6]. Moreover, we have reported the genetic clustering of clear cell RCCs based on arraycomparative genomic hybridization analysis and the association between genetic clustering on the one hand and clinicopathological tumor aggressiveness or patient outcome on the other [7]. With regard to epigenetic alterations, we have revealed that non-tumorous renal tissue obtained from patients with clear cell RCCs is at the precancerous stage, showing DNA hypo- and hypermethylation in multiple chromosomal regions [8], employing recently developed array-based technology [9], although precancerous conditions in the kidney have been rarely described because non-tumorous renal tissue shows no remarkable histological changes and is unassociated with chronic inflammation and persistent infection with viruses or other pathogenic microorganisms. We have proposed 2 possible scenarios: (a) genome-wide DNA methylation profiles of non-tumorous renal tissue at the precancerous stage are inherited by the corresponding clear cell RCCs developing in individual patients, and (b) DNA methylation alterations at the precancerous stage may be prone to further accumulation of genetic and epigenetic alterations during progression [9-11]. However, to our knowledge, the results of genome-wide DNA methylation analysis have never been reported for histological subtypes of renal tumors other than clear cell RCCs, such as papillary RCCs, chromophobe RCCs and oncocytomas, though the DNA methylation status of several tumor-related genes has been reported separately in such histological subtypes $[8,12,13]$.

In the present study, in order to clarify genome-wide DNA methylation profiles during multistage renal tumorigenesis, we performed bacterial artificial chromosome (BAC) array-based methylated $\mathrm{CpG}$ island amplification (BAMCA) [14-16] using a microarray of 4,361 BAC clones [17] for papillary RCCs, chromophobe RCCs and oncocytomas, and the corresponding non-tumorous renal tissue. DNA methylation profiles of patients with renal tumors of such histological subtypes were compared with those of patients with clear cell RCCs revealed by the same method.

\section{Materials and Methods}

Patients and Tissue Samples

Tumorous tissue and corresponding non-tumorous renal tissue samples were obtained at nephrectomy from 17 patients with primary renal tumors. These patients had not received preoperative treatment and had undergone nephrectomy between 1999 and 2006 at the National Cancer Center Hospital, Tokyo, Japan. The 17 primary renal tumors were histologically subclassified into 4 papillary RCCs including 2 type 1 papillary RCCs (T52 and T53) and 2 type 2 papillary RCCs (T54 and T55), 10 chromophobe RCCs (T56-T65) and 3 oncocytomas (T66-T68) in accordance with the World Health Organization classification (fig. 1a-d) [6]. Tumors in which almost the entire area showed a papillary or tubulopapillary architecture were classified as papillary RCCs, whereas clear cell RCCs in which only a minor component showed a papillary structure were not. The DNA methylation profiles of tumorous tissue and the corresponding non-tumorous renal tissue from these 17 patients were compared with those from 51 patients with clear cell RCCs (T1-T51; fig. 1e) for whom the results obtained by BAMCA had been reported previously [9]. All the patients gave their informed consent prior to inclusion in this study, which was approved by the Ethics Committee of the National Cancer Center, Tokyo, Japan.

\section{BAMCA Analysis}

High-molecular-weight DNA from fresh frozen tissue samples was extracted using phenol-chloroform, followed by dialysis. Because the DNA methylation status is known to be organ specific [18], the reference DNA for analysis of the developmental stages of renal tumors should be obtained from the kidney, and not from other organs or peripheral blood. Therefore, a mixture of normal renal tissue DNA obtained from 6 male patients and 3 female pa-

Fig. 1. Microscopic views (a-e, $\mathbf{k}-\mathbf{p})$ and scattergrams of the signal ratios (test signal/reference signal) obtained by BAMCA (f-j, $\mathbf{q}-\mathbf{v})$ in tumorous tissue $(\mathbf{a}-\mathbf{j})$ and non-tumorous renal tissue $(\mathbf{k}-\mathbf{o}, \mathbf{q}-$ $\mathbf{u}$ from patients with type 1 papillary $\operatorname{RCC}(\mathbf{a}, \mathbf{f}, \mathbf{k}, \mathbf{q})$, type 2 papillary RCC (b, g, I, r), chromophobe RCC (c, h, m, s), oncocytoma $(\mathbf{d}, \mathbf{i}, \mathbf{n}, \mathbf{t})$ and clear cell RCC $(\mathbf{e}, \mathbf{j}, \mathbf{o}, \mathbf{u})$, and normal renal tissue obtained from a patient without any renal tumor $(\mathbf{p}, \mathbf{v})$. In type 1 (a) and type 2 (b) papillary RCCs, chromophobe RCCs (c), oncocytomas (d) and clear cell RCCs (e), many BAC clones showed DNA hypo- or hypermethylation $(\mathbf{f}-\mathbf{j})$. Non-tumorous renal tissue obtained from patients with type $1(\mathbf{k})$ and type 2 papillary RCCs (I), chromophobe RCCs (m), oncocytomas (n) and clear cell RCCs (o) showed no histological changes in comparison with normal renal tissue (p) and could not be histologically discriminated from each other. Even in such non-tumorous renal tissue from patients with type $1(\mathbf{q})$ and type $2(\mathbf{r})$ papillary RCCs and clear cell RCCs (u), distinct DNA hypo- or hypermethylation was already evident when compared with normal renal tissue $(\mathbf{v})$. However, the numbers of BAC clones showing DNA hypo- or hypermethylation in non-tumorous renal tissue obtained from patients with chromophobe RCCs (s) and oncocytomas (t) were significantly smaller than those in non-tumorous renal tissue obtained from patients with clear cell RCCs (u) (table 1). 


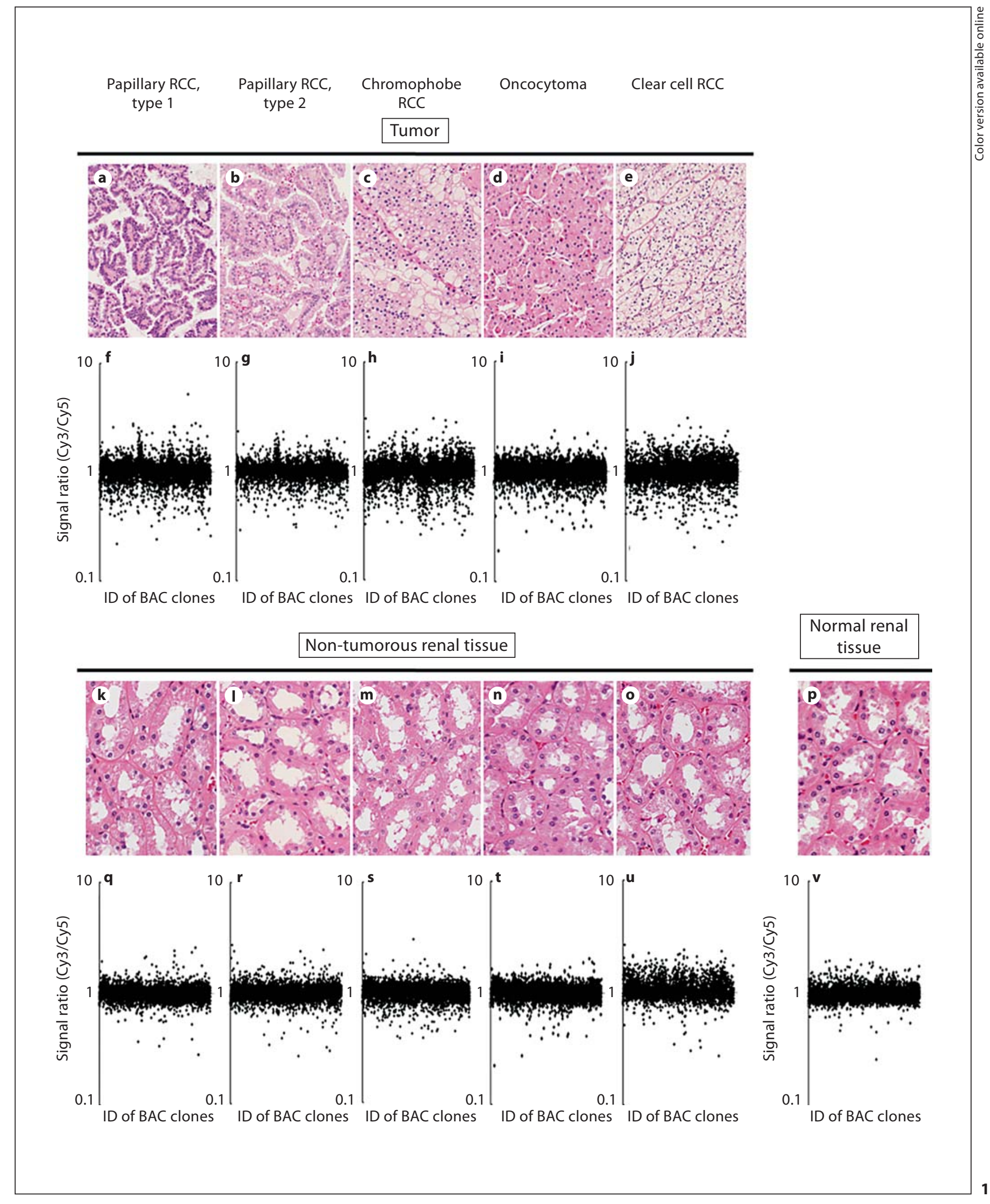

DNA Methylation Profiles in Renal

Pathobiology 2011;78:1-9 
Table 1. The average number of BAC clones showing DNA methylation alterations (DNA hypo- or hypermethylation) in tumorous tissue and non-tumorous renal tissue obtained from patients with renal tumors

\begin{tabular}{|c|c|c|c|c|}
\hline & \multicolumn{2}{|l|}{ Tumor } & \multicolumn{2}{|l|}{ Non-tumorous renal tissue } \\
\hline & $\begin{array}{l}\text { average number of BAC clones } \\
\text { showing DNA methylation alterations }\end{array}$ & $\mathrm{p}^{1}$ & $\begin{array}{l}\text { average number of BAC clones } \\
\text { showing DNA methylation alterations }\end{array}$ & $\mathrm{p}^{1}$ \\
\hline Papillary RCC ( $\mathrm{n}=4)$ & $400.5 \pm 249.6$ & 0.390 & $108.0 \pm 95.4$ & 0.173 \\
\hline Chromophobe RCC $(\mathrm{n}=10)$ & $334.4 \pm 139.7$ & 0.167 & $89.0 \pm 48.7$ & 0.041 \\
\hline Oncocytoma $(\mathrm{n}=3)$ & $266.7 \pm 205.7$ & 0.970 & $54.0 \pm 2.6$ & 0.028 \\
\hline Clear cell RCC $(n=51)$ & $265.3 \pm 150.5$ & - & $176.4 \pm 138.2$ & - \\
\hline
\end{tabular}

${ }^{1}$ Mann-Whitney U test, compared with patients with clear cell RCCs.

$\mathrm{p}$ values $<0.05$, which indicate significant differences, are italicized.

tients without any primary renal tumor was used as a reference for analyses of male and female test DNA samples, respectively.

The genome-wide DNA methylation status was analyzed by BAMCA using a custom-made array (MCG Whole Genome Array-4500) harboring 4,361 BAC clones throughout chromosomes $1-22$ and $X$ and $Y$ [17], as described previously [19, 20]. Briefly, $5-\mu \mathrm{g}$ aliquots of test or reference DNA were first digested with 100 units of the methylation-sensitive restriction enzyme SmaI and subsequently with 20 units of the methylation-insensitive $X m a I$. Adapters were ligated to XmaI-digested sticky ends, and PCR was performed with an adapter primer set. Test and reference PCR products were labeled by random priming with $\mathrm{Cy} 3$ and Cy5-dCTP (GE Healthcare, Little Chalfont, UK), respectively, using a BioPrime Array CGH Genomic Labeling System (Invitrogen, Carlsbad, Calif., USA) and precipitated together with ethanol in the presence of Cot-I DNA. The mixture was applied to array slides and incubated at $43^{\circ} \mathrm{C}$ for $72 \mathrm{~h}$. The arrays were scanned with a GenePix Personal 4100A (Axon Instruments, Foster City, Calif., USA) and analyzed using GenePix Pro 5.0 imaging software (Axon Instruments) and Acue 2 software (Mitsui Knowledge Industry, Tokyo, Japan). The signal ratios were normalized in each sample to make the mean signal ratios for all BAC clones 1.0. In accordance with previously described criteria [9], in the tumor and the corresponding non-tumorous renal tissue, DNA methylation status corresponding to a signal ratio of $<0.67$ and $>1.5$ was defined as DNA hypomethylation and DNA hypermethylation of each BAC clone compared with normal renal tissue, respectively. In our previous study, tumorous tissue and the corresponding non-tumorous renal tissue of 51 patients with clear cell RCCs (T1-T51) were analyzed by the same BAMCA method using the same array and reference DNA (fig. 1j) [9].

\section{Statistics}

Two-dimensional unsupervised hierarchical clustering analysis of the renal tumors with various histological subtypes and the $\mathrm{BAC}$ clones based on the signal ratios (test signal/reference signal) obtained by BAMCA were performed using the Expressionist software program (Genedata, Basel, Switzerland). Differences in the average number of BAC clones that showed DNA hypo- or hypermethylation between the histological subtypes of tumors were analyzed using the Mann-Whitney U test. BAC clones whose signal ratios differed significantly between chromophobe RCCs and oncocytomas were identified by the Wilcoxon test $(\mathrm{p}<0.01)$. Survival curves of patient groups were calculated by the KaplanMeier method, and the differences were compared by the log-rank test. Differences at $\mathrm{p}<0.05$ were considered significant.

\section{Results}

\section{Genome-Wide DNA Methylation Profiles of Renal Tumors}

Example scattergrams of the signal ratios (test signal/ reference signal) for tumorous tissue from each patient with type 1 papillary RCC, type 2 papillary RCC, chromophobe RCC and oncocytoma, respectively, are shown in figure $1 \mathrm{f}-\mathrm{i}$. The average numbers of BAC clones showing DNA hypo- or hypermethylation in papillary RCCs, chromophobe RCCs and oncocytomas were not significantly different from those in clear cell RCCs (table 1).

Figure 2 shows the results of 2-dimensional unsupervised hierarchical clustering based on the signal ratios obtained by BAMCA for 4 papillary RCCs (T52 and T55), 10 chromophobe RCCs (T56-T65), 3 oncocytomas (T66T68) and the previously examined 51 clear cell RCCs (T1T51). Two type 1 papillary RCCs (T52 and T53) and 2 type 2 papillary RCCs (T54 and T55) were clustered into the same subclasses and the 2 types of papillary RCCs were clustered into subclasses different from each other, and each accompanied clear cell RCCs.

All 10 chromophobe RCCs (T56-T65) and 3 oncocytomas (T66-T68) were clustered into the same subclass and excluded any tumor of other histological subtypes. On the other hand, the Wilcoxon test $(\mathrm{p}<0.01)$ revealed that the signal ratios of 21 BAC clones differed significantly between chromophobe RCCs $(n=10)$ and oncocy- 


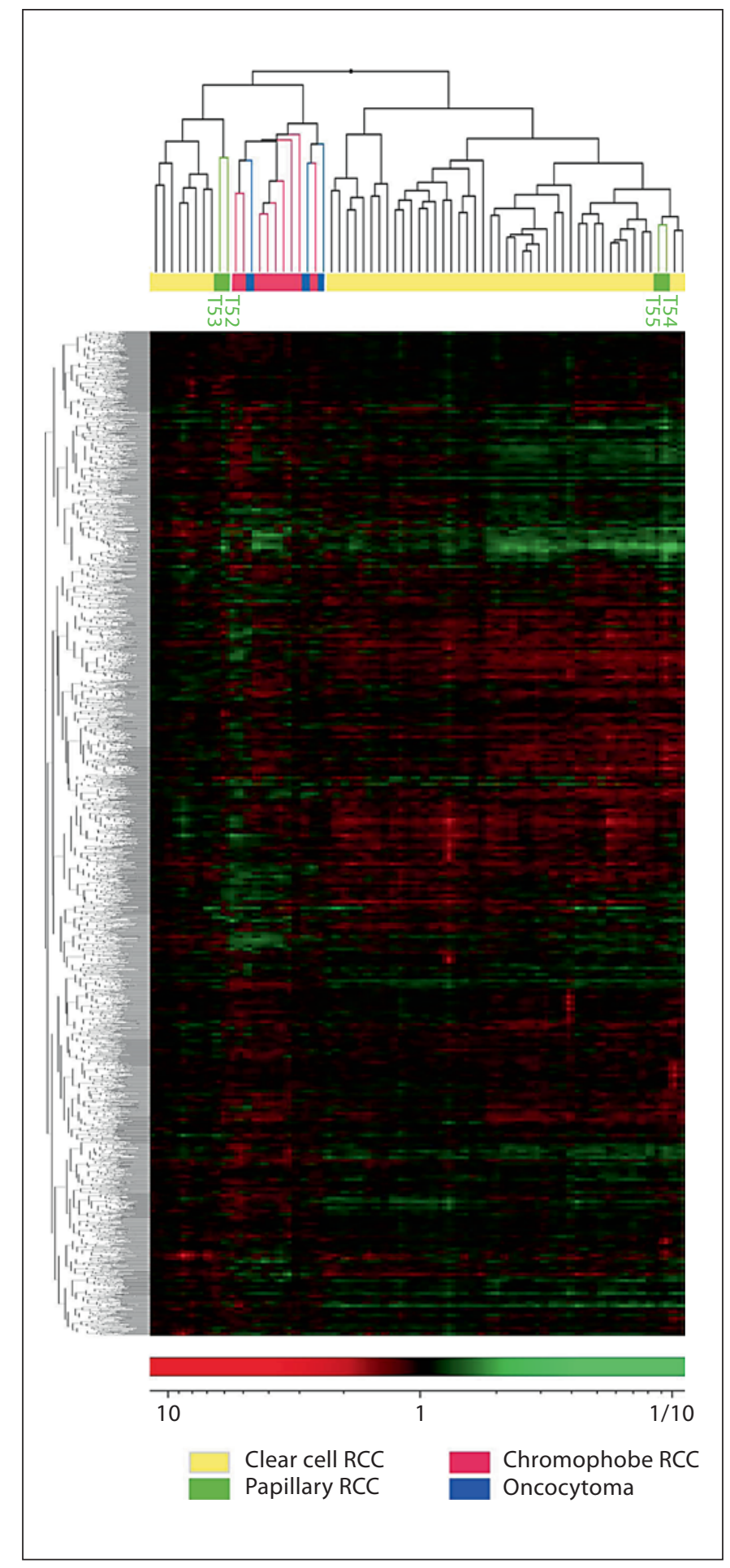

Fig. 2. Two-dimensional unsupervised hierarchical clustering analysis based on BAMCA data (signal ratios) for tumorous tissue obtained from patients with type 1 (T52 and T53) and type 2 (T54 and T55) papillary RCCs, chromophobe RCCs (T56-T65), oncocytomas (T66-T68) and clear cell RCCs (T1-T51). The signal ratio is shown as color range maps. The cluster trees for patients and BAC clones are shown at the top and left of the panel, respective- tomas $(\mathrm{n}=3)$. Figure 3 shows scattergrams of the signal ratios in chromophobe RCCs and oncocytomas for representative examples of the $21 \mathrm{BAC}$ clones. In all $21 \mathrm{BAC}$ clones, using the cutoff values of the signal ratios described in figure 3 and table 2, chromophobe RCCs in this cohort were discriminated from oncocytomas with a sensitivity and specificity of $100 \%$.

\section{Genome-Wide DNA Methylation Profiles of \\ Non-Tumorous Renal Tissue Obtained from Patients with Renal Tumors \\ In our previous study, many BAC clones showed DNA} hypo- or hypermethylation even in non-tumorous renal tissue obtained from patients with clear cell RCCs (fig. lu) when compared with normal renal tissue obtained from patients without any renal tumor (fig. 1v) [9], although non-tumorous renal tissue obtained from patients with clear cell RCCs (fig. 10) showed no histological changes in comparison with normal renal tissue (fig. 1p). Nontumorous renal tissue obtained from patients with papillary RCCs (fig. 1k, l), chromophobe RCCs (fig. $1 \mathrm{~m}$ ) and oncocytomas (fig. 1n) did not show any histological changes when compared with both non-tumorous renal tissue obtained from patients with clear cell RCCs (fig. 1o) and normal renal tissue (fig. 1p). Furthermore, there were no histological differences among non-tumorous renal tissue obtained from patients with papillary RCCs (fig. $1 \mathrm{k}, 1$ ), chromophobe RCCs (fig. $1 \mathrm{~m}$ ) and oncocytomas (fig. 1n). However, the average numbers of BAC clones showing DNA hypo- or hypermethylation in non-tumorous renal tissue obtained from patients with chromophobe RCCs and oncocytomas were significantly smaller than the average number in non-tumorous renal tissue obtained from patients with clear cell RCCs (table 1).

A histogram showing the numbers of BAC clones with DNA hypo- and hypermethylation in non-tumorous renal tissue from all 68 patients with renal tumors is shown in figure $4 \mathrm{a}$. Biphasic accumulation of DNA methylation alterations was evident, with a trough of $250 \mathrm{BAC}$ clones in non-tumorous renal tissue. Thus, the 68 patients were divided into 2 groups according to the number of BAC

ly. Two type 1 papillary RCCs (T52 and T53) and 2 type 2 papillary RCCs (T54 and T55) were each clustered into the same subclasses. Type 1 and type 2 papillary RCCs were clustered into subclasses different from each other, and each was accompanied by clear cell RCCs. All 10 chromophobe RCCs and 3 oncocytomas were clustered into the same subclass, which did not include any tumor of other histological subtypes. 
Fig. 3. Scattergrams of the signal ratios in chromophobe RCCs (Chr, T56-T65) and 3 oncocytomas (Onc, T66-T68) for representative BAC clones, RP11-140B17, RP11678G14 and RP11-404P16. Using the cutoff values (CV) described in each panel, chromophobe RCCs were discriminated from oncocytomas with a sensitivity and specificity of $100 \%$.

Fig. 4. a Histogram showing the number of BAC clones with DNA hypo- or hypermethylation in non-tumorous renal tissue obtained from patients with papillary RCCs, chromophobe RCCs, oncocytomas and clear cell RCCs. Biphasic accumulation of DNA methylation alterations with a trough of 250 BAC clones (arrow) was observed in non-tumorous renal tissue. b Kaplan-Meier survival curves of patients with papillary RCCs, chromophobe RCCs, oncocytomas and clear cell RCCs. The period covered ranged from 63 to 2,801 days (mean 1,612). The recurrence-free survival rate of patients showing DNA hypo- or hypermethylation on $\geq 250$ BAC clones in their non-tumorous renal tissue $(n=16$, black line) was significantly lower than that of patients showing DNA hypo- or hypermethylation on $<250 \mathrm{BAC}$ clones in their non-tumorous renal tissue $(n=50$, gray line; $\mathrm{p}=0.0204$ ).
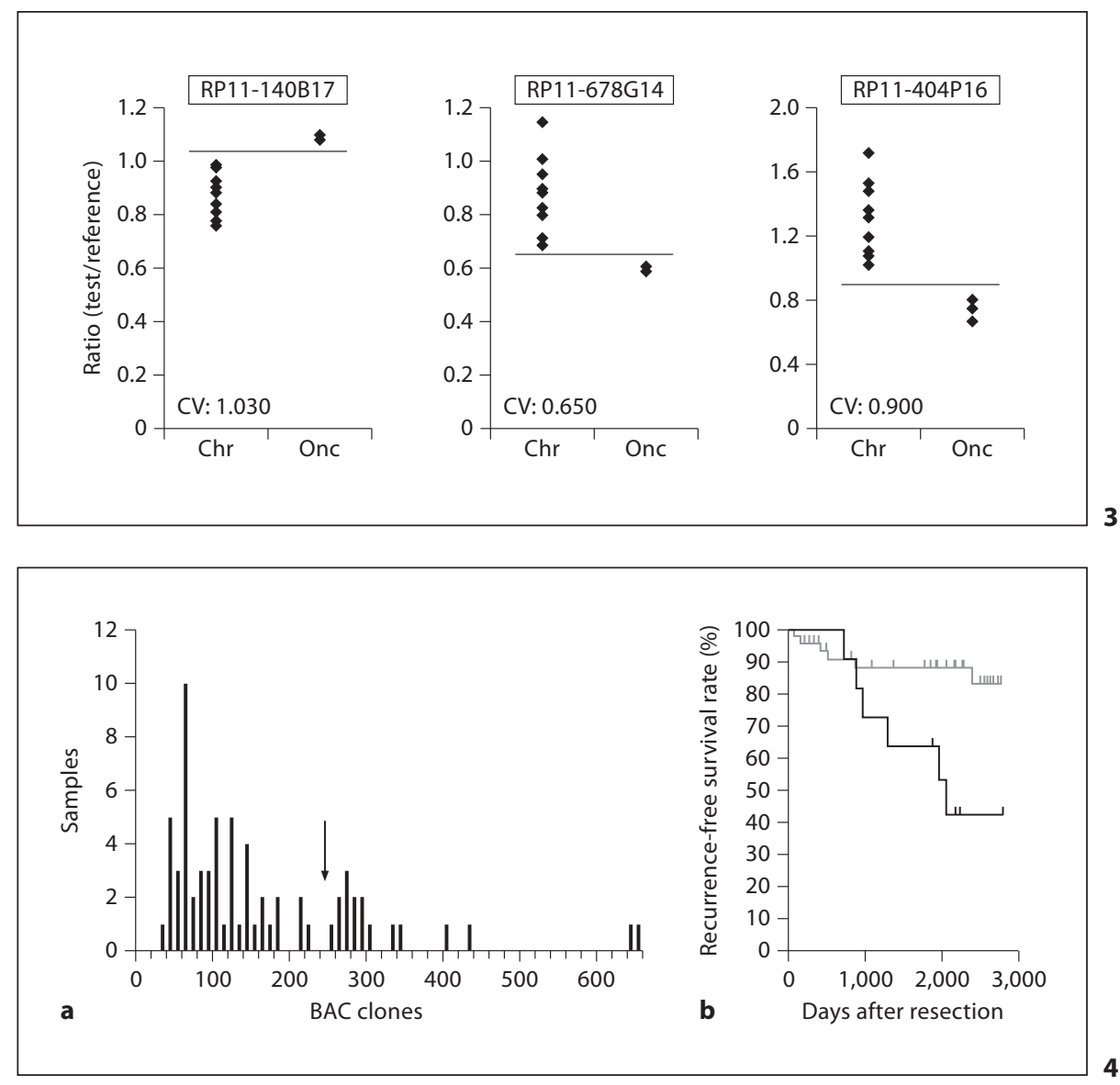

clones showing DNA hypo- or hypermethylation in their non-tumorous renal tissue ( $\geq 250$ BAC clones vs. $<250$ BAC clones). Figure $4 \mathrm{~b}$ shows the Kaplan-Meier survival curves of 66 patients who underwent curative resection of their renal tumors. The period covered ranged from 63 to 2,801 days (mean 1,612). The recurrence-free survival rate of patients showing DNA hypo- or hypermethylation on $\geq 250$ BAC clones in their non-tumorous renal tissue was significantly lower than that of patients showing DNA hypo- or hypermethylation on $<250$ BAC clones $(\mathrm{p}=0.0204$; fig. $4 \mathrm{~b})$.

\section{Discussion}

In tumors of many organs, an association between specific DNA methylation profiles and various histological subtypes has been reported [21,22]. Such an association may reflect an epigenetic pathway of tumorigenesis, which is specific to each histological subtype. Although various histological subtypes of tumors occur in the kidney, to our knowledge, there has been no reported genome-wide DNA methylation analysis of such histological subtypes other than clear cell RCC.

Many researchers in the field of cancer epigenetics have used promoter arrays to identify the genes that are methylated in cancer cells [23-25]. However, the promoter regions of specific genes are not the only target of DNA methylation alterations in human cancers. DNA methylation status in genomic regions that do not directly participate in gene silencing, such as the edges of $\mathrm{CpG}$ islands, may be altered at the precancerous stage before the alterations of the promoter regions themselves occur [26]. Genomic regions in which DNA hypomethylation affects chromosomal instability may not be contained in promoter arrays. Moreover, aberrant DNA methylation of large regions of chromosomes, which are regulated in a coordinated manner in human cancers due to a process of long-range epigenetic silencing, has recently attracted attention [27]. Therefore, we used a custom-made BAC 
Table 2. Twenty-one BAC clones which were able to discriminate chromophobe RCCs (Chr) from oncocytomas (Onc)

\begin{tabular}{|c|c|c|c|}
\hline BAC clone ID & Location & $\begin{array}{l}\text { Cutoff } \\
\text { value }\end{array}$ & $\begin{array}{l}\text { DNA meth- } \\
\text { ylation status }\end{array}$ \\
\hline RP11-201O14 & $1 \mathrm{p} 34.3-1 \mathrm{p} 36.13$ & 0.950 & $\mathrm{Chr}<\mathrm{Onc}$ \\
\hline RP11-89O18 & $1 \mathrm{p} 33-1 \mathrm{p} 34.2$ & 1.070 & $\mathrm{Chr}<\mathrm{Onc}$ \\
\hline RP11-542D13 & $2 \mathrm{q} 11.1-2 \mathrm{q} 11.2$ & 0.850 & $\mathrm{Chr}<\mathrm{Onc}$ \\
\hline RP11-124O2 & $3 \mathrm{p} 21.2$ & 0.610 & $\mathrm{Chr}>\mathrm{Onc}$ \\
\hline RP11-745L2 & $3 \mathrm{q} 13.13 \mathrm{~d}$ & 1.135 & $\mathrm{Chr}<\mathrm{Onc}$ \\
\hline RP11-89F1 & $5 q 32$ & 0.950 & $\mathrm{Chr}<\mathrm{Onc}$ \\
\hline RP11-79J23 & $6 \mathrm{p} 21.2-6 \mathrm{p} 21.3$ & 1.070 & $\mathrm{Chr}<\mathrm{Onc}$ \\
\hline RP11-75C8 & $6 q 21-6 q 22.1$ & 0.920 & $\mathrm{Chr}<\mathrm{Onc}$ \\
\hline RP11-10D8 & $7 \mathrm{q} 22.1$ & 0.780 & $\mathrm{Chr}>\mathrm{Onc}$ \\
\hline RP11-140B17 & $10 \mathrm{q} 25.3-10 \mathrm{q} 26.13$ & 1.030 & $\mathrm{Chr}<\mathrm{Onc}$ \\
\hline RP11-196E1 & $11 q 23$ & 0.910 & Chr $>$ Onc \\
\hline RP11-170D9 & $14 \mathrm{q} 11.2-14 \mathrm{q} 12$ & 0.920 & $\mathrm{Chr}>\mathrm{Onc}$ \\
\hline RP11-91J13 & $14 \mathrm{q} 23$ & 1.000 & Chr $>$ Onc \\
\hline RP11-397B22 & $16 \mathrm{p} 13.3 \mathrm{a}$ & 0.960 & $\mathrm{Chr}>\mathrm{Onc}$ \\
\hline RP11-122P17 & $16 q 24$ & 0.850 & Chr $>$ Onc \\
\hline RP11-798B19 & $19 \mathrm{p}$ & 1.050 & $\mathrm{Chr}>\mathrm{Onc}$ \\
\hline RP11-678G14 & $19 \mathrm{p} 12 \mathrm{~b}-19 \mathrm{p} 12 \mathrm{c}$ & 0.650 & Chr $>$ Onc \\
\hline RP11-46I12 & $19 q 12-19 q 13.1$ & 0.650 & $\mathrm{Chr}>\mathrm{Onc}$ \\
\hline RP11-446K10 & $19 q 13.1-19 q 13$ & 0.950 & Chr $>$ Onc \\
\hline RP11-10D18 & $20 q 13.1-20 q 13.2$ & 0.720 & $\mathrm{Chr}>\mathrm{Onc}$ \\
\hline RP11-404P16 & $\mathrm{Xp} 11.2-\mathrm{Xp} 11.3$ & 0.900 & $\mathrm{Chr}>\mathrm{Onc}$ \\
\hline
\end{tabular}

${ }^{1} \mathrm{Chr}<\mathrm{Onc}=$ when the signal ratio was lower than the cutoff value, the tissue sample was considered to have originated from chromophobe RCC; $\mathrm{Chr}>\mathrm{Onc}=$ when the signal ratio was higher than the cutoff value, the tissue sample was considered to have originated from chromophobe RCC.

array [17] that may be suitable not for focusing on specific promoter regions, but for overviewing the DNA methylation status of individual large regions among all chromosomes.

Since microscopic observation frequently revealed a papillary RCC component associated with the concomitant clear cell RCC component in a single renal tumor, papillary RCCs and subclasses of clear cell RCCs may have been grouped into the same subclass in the present unsupervised hierarchical clustering (fig. 2). First, based simply on cytologic and histologic criteria, papillary RCCs were divided into 2 morphologic groups, type 1 and type 2. Type 1 papillary RCCs consist of papillae covered with a single or double layer of small cuboid cells with scanty cytoplasm, and type 2 papillary RCCs consist of papillae covered by large eosinophilic cells arranged in an irregular or pseudo-stratified manner [6]. Although type 2 papillary RCC is frequently at an advanced stage at initial diagnosis, thus resulting in poor patient survival [28], only a small number of molecular differences between type 1 and type 2 papillary RCCs, such as the level of expression of vascular endothelial growth factor receptor and copy number alterations on chromosomes $1 \mathrm{p}, 3 \mathrm{p}$, $9 p$ and 17, have been reported to date [29]. The present results (fig. 2) indicate that genome-wide DNA methylation profiles may explain the differences in background characteristics between type 1 and type 2 papillary RCCs, although further confirmation in a larger cohort will be needed.

In the present unsupervised hierarchical clustering based on BAMCA data, chromophobe RCCs and oncocytomas formed a subclass by themselves (fig. 2). Histopathological observations have underlined the similarities between chromophobe RCCs and oncocytomas. Since both of these neoplasms consist of tumor cells with abundant eosinophilic cytoplasm and mainly show a solid structure [6], differential diagnosis between them frequently becomes difficult even for experienced pathologists. Both chromophobe RCCs and oncocytomas have been described in patients with Birt-Hogg-Dubé syndrome, which is characterized by cutaneous fibrofolliculomas, renal tumors, pulmonary cysts and spontaneous pneumothorax. Moreover, such patients sometimes develop so-called hybrid oncocytic tumors with histological features similar to both chromophobe RCCs and oncocytomas [30]. On the other hand, the genetic status of chromophobe RCCs and oncocytomas differs markedly: copy number alterations on various chromosomes are frequent in chromophobe RCCs, but are rare in oncocytomas [31]. The present results indicate that similarities of genome-wide DNA methylation profiles may epigenetically cover the genetic differences between chromophobe RCCs and oncocytomas and may be able to explain the phenotypic similarities of these tumors. On the other hand, regional DNA methylation alterations on the 21 $\mathrm{BAC}$ clones were able to discriminate chromophobe RCCs and oncocytomas (fig. 3; table 2). Even though the overall epigenetic pathway of tumorigenesis may be shared by chromophobe RCCs and oncocytomas, there may be target chromosomal regions of DNA methylation alterations that are specific to each neoplasm. In addition to copy number status, the DNA methylation status in such chromosomal regions may become a hallmark for differential diagnosis of these morphologically similar tumors.

Surprisingly, the DNA methylation status (the number of BAC clones showing DNA hypo- or hypermethylation) of non-tumorous renal tissue obtained from chro- 
mophobe RCCs and oncocytomas was significantly different from that of clear cell RCCs, suggesting that histological subtype-specific DNA methylation alterations have already occurred, even in apparently normal renal tissue (table 1). Although we analyzed samples of non-tumorous renal cortex tissue as well as tumorous tissue using normal renal cortex tissue as a reference for the comparison of all histological subtypes (table 1), chromophobe RCCs and oncocytomas are considered to be derived from the intercalated cells of the collecting duct. We also examined DNA methylation status in non-tumorous renal medulla tissue obtained from patients with chromophobe RCCs using a mixture of normal renal medulla DNA as a reference. The numbers of BAC clones showing DNA methylation alterations in renal medulla tissue did not differ significantly from those in renal cortex tissue obtained from individual patients with chromophobe RCCs (online supplementary table 1, www. karger.com/doi/10.1159/000322072). Therefore, we were able to observe differences of DNA methylation status between non-tumorous renal tissue from patients with chromophobe RCCs and that from patients with clear cell RCCs even when we used tissue samples of the renal medulla and cortex, which are the tissues of origin of chromophobe RCCs and clear cell RCCs, respectively. It is possible that the DNA methylation status of non-tumorous renal tissue obtained from patients with papillary RCCs was not different from that of clear cell RCCs (table 1), because papillary RCCs themselves showed DNA methylation profiles similar to those of clear cell RCCs (fig. 2).

It is known that patients with chromophobe RCCs and oncocytomas generally show a more favorable outcome than patients with clear cell RCCs [32]. Since patients with chromophobe RCCs and oncocytomas showed DNA methylation alterations in non-tumorous renal tissue that differed from those of patients with more aggressive clear cell RCCs (table 1), we evaluated the correlation between the DNA methylation status of non-tumorous renal tissue and patient outcome. Surprisingly, patients with accumulation of DNA methylation (DNA hypo- or hypermethylation on $\geq 250$ BAC clones) in their non-tumorous renal tissue showed a poorer outcome than patients without such accumulation (DNA hypo- or hypermethylation on $<250$ BAC clones; fig. 4b). Although one cannot easily conclude that DNA methylation alterations in non-tumorous renal tissue are correlated with histological subtype (chromophobe RCCs and oncocytomas vs. clear cell RCCs) or patient outcome (favorable outcome vs. poorer outcome), or both, the present study including various histological subtypes indicated that DNA methylation status was not simply altered in precancerous conditions, but that significant DNA methylation profiles determining the histological subtypes of future developing renal tumors and/or patient outcome are already established at the precancerous stage.

\section{Acknowledgements}

This study was supported by a Grant-in-Aid for the Third Term Comprehensive 10-Year Strategy for Cancer Control from the Ministry of Health, Labor and Welfare of Japan, a Grant-inAid for Cancer Research from the Ministry of Health, Labor and Welfare of Japan, a grant from the New Energy and Industrial Technology Development Organization, and the Program for Promotion of Fundamental Studies in Health Sciences of the National Institute of Biomedical Innovation.

\section{References}

1 Jones PA, Baylin SB: The fundamental role of epigenetic events in cancer. Nat Rev Genet 2002;3:415-428.

-2 Eden A, Gaudet F, Waghmare A, Jaenisch R: Chromosomal instability and tumors promoted by DNA hypomethylation. Science 2003;300:455.

-3 Baylin SB, Ohm JE: Epigenetic gene silencing in cancer - a mechanism for early oncogenic pathway addiction? Nat Rev Cancer 2006;6: 107-116.
4 Kanai Y, Hirohashi S: Alterations of DNA methylation associated with abnormalities of DNA methyltransferases in human cancers during transition from a precancerous to a malignant state. Carcinogenesis 2007;28: 2434-2442.

5 Kanai Y: Alterations of DNA methylation and clinicopathological diversity of human cancers. Pathol Int 2008;58:544-558.

6 Eble JN, Togashi K, Pisani P: Renal cell carcinoma; in Eble JN, Sauter G, Epsterin JI (ed): World Health Organzation Classification of Tumours. Pathology and Genetics. Tumours of the Urinary System and Male Genital Organs. Lyon, IARC Press, 2008, pp 10-43.
7 Arai E, Ushijima S, Tsuda H, Fujimoto H, Hosoda F, Shibata T, Kondo T, Imoto I, Inazawa J, Hirohashi S, Kanai Y: Genetic clustering of clear cell renal cell carcinoma based on array-CGH: its association with DNA methylation alteration and patient outcome. Clin Cancer Res 2008;14:5531-5539.

8 Arai E, Kanai Y, Ushijima S, Fujimoto H, Mukai K, Hirohashi S: Regional DNA hypermethylation and DNA methyltransferase (DNMT) 1 protein overexpression in both renal tumors and corresponding nontumorous renal tissues. Int J Cancer 2006;119:288296. 
-9 Arai E, Ushijima S, Fujimoto H, Hosoda F, Shibata T, Kondo T, Yokoi S, Imoto I, Inazawa J, Hirohashi S, Kanai Y: Genome-wide DNA methylation profiles in both precancerous conditions and clear cell renal cell carcinomas are correlated with malignant potential and patient outcome. Carcinogenesis 2009;30:214-221.

10 Arai E, Kanai Y: DNA methylation profiles in precancerous tissue and cancers: carcinogenetic risk estimation and prognostication based on DNA methylation status. Epigenomics 2010;2:476-481.

11 Kanai Y: Genome-wide DNA methylation profiles in precancerous conditions and cancers. Cancer Sci 2010;101:36-45

-12 Battagli C, Uzzo RG, Dulaimi E, Ibanez de Caceres I, Krassenstein R, Al-Saleem T, Greenberg RE, Cairns P: Promoter hypermethylation of tumor suppressor genes in urine from kidney cancer patients. Cancer Res 2003;63:8695-8699.

-13 Dulaimi E, Ibanez de Caceres I, Uzzo RG, AlSaleem T, Greenberg RE, Polascik TJ, Babb JS, Grezle WE, Cairns P: Promoter hypermethylation profile of kidney cancer. Clin Cancer Res 2004;10:3972-3979.

- 14 Misawa A, Inoue J, Sugino Y, Hosoi H, Sugimoto T, Hosoda F, Ohki M, Imoto I, Inazawa $\mathrm{J}$ : Methylation-associated silencing of the nuclear receptor 1 II gene in advanced-type neuroblastomas, identified by bacterial artificial chromosome array-based methylated CpG island amplification. Cancer Res 2005; 65:10233-10242.

-15 Sugino Y, Misawa A, Inoue J, Kitagawa M, Hosoi $\mathrm{H}$, Sugimoto T, Imoto I, Inazawa J: Epigenetic silencing of prostaglandin E receptor 2 (PTGER2) is associated with progression of neuroblastomas. Oncogene 2007; 26:7401-7413.

-16 Tanaka K, Imoto I, Inoue J, Kozaki K, Tsuda H, Shimada Y, Aiko S, Yoshizumi Y, Iwai T, Kawano T, Inazawa J: Frequent methylationassociated silencing of a candidate tumorsuppressor, CRABP1, in esophageal squamous-cell carcinoma. Oncogene 2007;26: 6456-6468.
17 Inazawa J, Inoue J, Imoto I: Comparative genomic hybridization (CGH)-arrays pave the way for identification of novel cancer-related genes. Cancer Sci 2004;95:559-563.

18 Illingworth R, Kerr A, Desousa D, Jorgensen $\mathrm{H}$, Jorgensen H, Ellis P, Stalker J, Jackson D, Clee C, Plumb R, Rogers J, Humphray S, Cox T, Langford C, Bird A: A novel CpG island set identifies tissue-specific methylation at developmental gene loci. PLoS Biol 2008;6:22.

19 Arai E, Ushijima S, Gotoh M, Ojima H, Kosuge T, Hosoda F, Shibata T, Kondo T, Yokoi S, Imoto I, Inazawa J, Hirohashi S, Kanai Y: Genome-wide DNA methylation profiles in liver tissue at the precancerous stage and in hepatocellular carcinoma. Int J Cancer 2009; 125:2854-2862.

20 Nishiyama N, Arai E, Chihara Y, Fujimoto H, Hosoda F, Shibata T, Kondo T, Tsukamoto T, Yokoi S, Imoto I, Inazawa J, Hirohashi S, Kanai Y: Genome-wide DNA methylation profiles in urothelial carcinomas and urothelia at the precancerous stage. Cancer Sci 2010;101:231-240

21 Kaneuchi M, Sasaki M, Tanaka Y, Shiina H, Verma M, Ebina Y, Nomura E, Yamamoto R, Sakuragi N, Dahiya R: Expression and methylation status of 14-3-3 sigma gene can characterize the different histological features of ovarian cancer. Biochem Biophys Res Commun 2004;316:1156-1162.

22 Oue N, Oshimo Y, Nakayama H, Ito R, Yoshida K, Matsusaki K, Yasui W: DNA methylation of multiple genes in gastric carcinoma: association with histological type and CpG island methylator phenotype. Cancer Sci 2003;94:901-905.

-23 Estecio MR, Yan PS, Ibrahim AE, Tellez CS, Shen L, Huang TH, Issa JP: High-throughput methylation profiling by MCA coupled to CpG island microarray. Genome Res 2007; 17:1529-1536.

24 Jacinto FV, Ballestar E, Ropero S, Esteller M: Discovery of epigenetically silenced genes by methylated DNA immunoprecipitation in colon cancer cells. Cancer Res 2007;67: 11481-11486.
25 Nielander I, Bug S, Richter J, Giefing M, Martin-Subero JI, Siebert R: Combining array-based approaches for the identification of candidate tumor suppressor loci in mature lymphoid neoplasms. APMIS 2007;115: 1107-1134.

26 Maekita T, Nakazawa K, Mihara M, Nakajima T, Yanaoka K, Iguchi M, Arii K, Kaneda A, Tsukamoto T, Tatematsu M, Tamura G, Saito D, Sugimura T, Ichinose M, Ushijima T: High levels of aberrant DNA methylation in Helicobacter pylori-infected gastric mucosae and its possible association with gastric cancer risk. Clin Cancer Res 2006;12:989995.

-27 Frigola J, Song J, Stirzaker C, Hinshelwood RA, Peinado MA, Clark SJ: Epigenetic remodeling in colorectal cancer results in coordinate gene suppression across an entire chromosome band. Nat Genet 2006;38:540549.

-28 Waldert M, Haitel A, Marberger M, Katzenbeisser D, Ozsoy M, Stadler E, Remzi M: Comparison of type I and II papillary renal cell carcinoma (RCC) and clear cell RCC. BJU Int 2008;102:1381-1384.

29 Klatte T, Pantuck AJ, Said JW, Seligson DB, Rao NP, LaRochelle JC, Shuch B, Zisman A, Kabbinavar FF, Belldegrun AS: Cytogenetic and molecular tumor profiling for type 1 and type 2 papillary renal cell carcinoma. Clin Cancer Res 2009;15:1162-1169.

30 Adley BP, Smith ND, Nayar R, Yang XJ: BirtHogg-Dubé syndrome: clinicopathologic findings and genetic alterations. Arch Pathol Lab Med 2006;130:1865-1870.

-31 Monzon FA, Hagenkord JM, Lyons-Weiler MA, Balani JP, Parwani AV, Sciulli CM, Chandran UR, Bastacky SI, Dhir R: Whole genome SNP arrays as a potential diagnostic tool for the detection of characteristic chromosomal aberrations in renal epithelial tumors. Mod Pathol 2008;21:599-608.

32 Cheville JC, Lohse CM, Zincke H, Weaver $\mathrm{AL}$, Blute ML: Comparisons of outcome and prognostic features among histologic subtypes of renal cell carcinoma. Am J Surg Pathol 2003;27:612-624. 